\title{
Design and calibration of pinch force measurement using strain gauge for post-stroke patients
}

\begin{abstract}
Two fingers strength is an indicative measurement of pinch impairment. Conventionally, Fugl Meyer Upper Extremity Assessment (FMA-UE) is the primary standard to measure pinch strength of post-stroke survivors. In literature, the evaluation method performed by the therapist is subjective and exposed to inter-rater and intra-rater reliabilities. Recently, force-sensing resistors were implemented to measure two fingers force, but these sensors are subjected to nonlinearity, high hysteresis, and voltage drift. This paper presents a design of pinch force measurement based on the strain gauge. The pinch sensor was calibrated within a range of between $0 \mathrm{~N}$ to $50 \mathrm{~N}$ over a pinching length of $20 \mathrm{~mm}$ with a linearity error of $0.0123 \%$ and hysteresis of $0.513 \%$ during the loading and unloading process. The voltage drift has an average of $0.24 \%$ over 20 minutes. The pinch force measurement system reveals an objective pinch force measurements in evaluating the rehabilitation progress of post-stroke patients.
\end{abstract}

Keyword: Post-stroke rehabilitation; Fugl Meyer; Pinch force; Objective assessment; Strain gauge; Arduino Due 